\title{
A Simulation for Parameters Extraction of Double-Layer Shielded Power Cable using FEA
}

\author{
$1^{\text {st }}$ Douglas Nascimento* \\ University of Zielona Góra \\ University of Twente \\ Zielona Góra, Poland \\ eng.douglas.a@ieee.org \\ $4^{\text {th }}$ Amr Madi \\ University of Zielona Góra \\ University of Twente \\ Zielona Góra, Poland \\ amr.madi@ieee.org
}

\author{
$2^{\text {nd }}$ Robert Smolenski \\ Instytut Automatyki, \\ Elektroniki i Elektrotechniki \\ University of Zielona Góra \\ Zielona Góra, Poland \\ r.smolenski@iee.uz.zgora.pl \\ $5^{\text {th }}$ Muhammad Alamsyah \\ Leibniz University Hannover \\ University of Nottingham \\ Hannover, Germany \\ alamsyah@geml.uni-hannover.de
}

\author{
$3^{\text {rd }}$ Hermes Loschi \\ University of Zielona Góra \\ University of Twente \\ University of Nottingham \\ Zielona Góra, Poland \\ eng.hermes.loschi@ieee.org \\ $6^{\text {th }}$ Francinei Vieira \\ Leibniz University Hannover \\ University of Nottingham \\ Hannover, Germany \\ vieira@geml.uni-hannover.de
}

\begin{abstract}
This paper addresses a preliminary research on numerical simulation method of an double-layer shielded and low voltage power cable for electric vehicle powertrain using Finite Element Analysis (FEA) and Simulation Program with Integrated Circuit Emphasis (SPICE) as software tools. Simulating this detailed method through FEA for only one transmission line cell, it was obtained the transmission lines parameters and the magnetic flux density of the cable. Then, its length is extended by using transmission lines theory and the frequency response of the cable was gathered as an evaluation of the current method implemented in SPICE. Thus, those analyses can provide a faster and better overview concerning the prediction of cable's electromagnetic (EM) shielding behavior in order to avoid Electromagnetic Compatibility (EMC) compliance issues in the early-stage development of vehicles.
\end{abstract}

Index Terms-Electromagnetic Compatibility, FEA, SPICE, Cables, Electric Vehicle.

\section{INTRODUCTION}

The electrical environment generates electromagnetic interference (EMI) among the vehicular electric powertrain components [1]. This imposes serious issues concerning electromagnetic compatibility (EMC) in which automotive manufacturers must withstand the standards [2].

Since the cables figure as one of the most susceptible elements of the vehicular electrical powertrain [3], [4] - due to stray components, induced currents coupled within the cable and harnesses acting as antennas, they must be addressed in a way to avoid EMC issues, e.g. either by using EMI filters, upgrading the grounding system or changing the cable design or layout within the electric vehicle (EV) [1].

Despite the possibility to overcome EMC issues in other environments by applying switching techniques [5] or adding

This paper has received funding from the European Union's Horizon 2020 research and innovation programme under the Marie Skłodowska-Curie grant agreement No.812753 and No.812391.

*Corresponding author: eng.douglas.a@ieee.org (Douglas Nascimento).
EMI filters, the space in a vehicular environment is quite limited [6] and integrating another component to perform those actions is not an option. In addition, the ground system is restricted to the vehicle metallic body. Thus, it only remains the improvement of cable design or layout. Considering of the fixed layout preset given the vehicle geometry, usually the cable design is the only possible option of improvement, as shown by [7].

Taking in account that there are bottlenecks addressing modeling methods of double-layer shielding power cables [8], this paper proposes the modeling of cable using FEA, based on [9]-[11], with high detailing levels and making use of transmission lines theory to speed up the early-phase development of EVs regarding shielding analysis. As contribution, it can reduce the computational overload specifying the model, with high detail levels, to design of $1 \mathrm{~cm}$-long $(1$ cell) vehicular electric powertrain cable and to extend it up to 100 cells by using transmission lines theory. At last it is analyzed the cable frequency response through SPICE (Simulation Program with Integrated Circuit Emphasis) as evaluation of the cable design.

\section{TRANSMisSion Line THEORY AND \\ Electromagnetics Applied to Coaxial Cables}

The transmission line can then be described as a cascade connected network chain composed of an infinitesimal value of differential lengths (segments), $\Delta l$, which is often taken as $1 / 10$ of the highest frequency wavelength in operation. The equivalent circuit for the segments of line is shown in Fig. 1. It consists of circuit elements R. $\Delta l$ (given as $\Omega / \mathrm{m}$ ), L. $\Delta l$ $(\mathrm{H} / \mathrm{m})$, C. $\Delta l(\mathrm{~F} / \mathrm{m})$ and G. $\Delta l(\mathrm{~S} / \mathrm{m})[12],[13]$.

By using Kirchhoff's Law the transmission line equations (telegraph equations) can be obtained for the segment cell considered and, thus, defining the distributed parameters of modeling. These parameters are used to calculate cable overall 


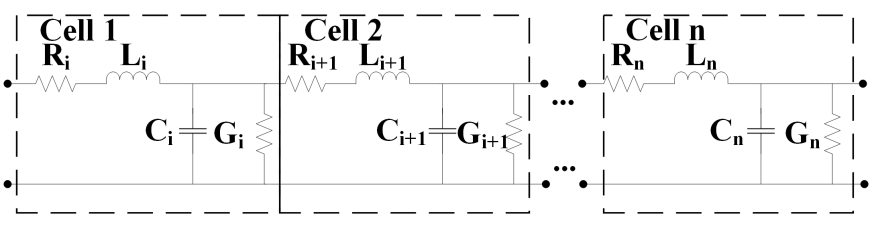

Fig. 1. Equivalent circuit network for distributed parameter divided by cells of $\Delta l$. Index $i$ denotes the number of the cell and, $n$ means the nth cell.

efficiency, characteristic impedance, and propagation characteristics, etc [13].

The propagation constant $(\gamma)$ is given by $\gamma=\alpha+j \beta$, where attenuation constant can be write as $\alpha=R+j \omega L$ and the phase constant is obtained by $\beta=G+j \omega C$ [12]. The term $\omega$ is the angular frequency and it is given by $\omega=2 \pi f$, with $f$ being the frequency of the study. Assuming the low frequency and short cable length used in this is study, $\gamma$ can be reduced to:

$$
\gamma=\sqrt{L C}
$$

Another parameter that must be drained attention is the reflection coefficient $(\Gamma)$ given by [14],

$$
\Gamma=\frac{Z_{L}-Z_{0}}{Z_{L}+Z_{0}}
$$

where $Z_{L}$ is the load impedance and $Z_{0}$ is the characteristic impedance of the transmission line. This parameter $\Gamma$ gives information about reflection by any impedance mismatching between the line and the load.

\section{A. Electromagnetic Shielding}

The magnetic flux density $B$, defined as $B=\mu H$, where $\mu=\mu_{r} \times \mu_{0}$ - which states that $B$ is material dependent and $H$ is the magnetic field intensity. This is can be applied to investigate the magnetic force lines passing through the material area [14].

Despite being one important requirement in cables design, either the analysis of shielding efficiency or stray components are not the objective of this study and for more details refer [12].

\section{B. Network Frequency Response}

The wavelength equation of the wave propagation is given by [12],

$$
\lambda=\frac{c}{f_{H O}}
$$

in which $c$ is the light speed with $c=2.9979 \times 10^{8} \mathrm{~m} / \mathrm{s}$ and $f_{H 0}$ is the maximum operation frequency considered in the study. Thus considering an arrange of capacitor and inductor operating in resonance, it is possible to infer the frequency operation range based on,

$$
f_{r}=\frac{1}{2 \pi \sqrt{L \times C}}
$$

The characteristic impedance is given by:

$$
Z_{0}=\sqrt{\frac{R+j \omega L}{G+j \omega C}}
$$

Due to the frequency range (up to $100 \mathrm{kHz}$ ) and length of the cable $(1 \mathrm{~m})$ adopted the values of $\mathrm{R}$ and $\mathrm{G}$ can be disregarded once they are very small,

$$
Z_{0}=\sqrt{\frac{L}{C}}
$$

Therefore, the methodology of this work involves the frequency response analysis is discussed in Section III and the simulation through SPICE to validate the study in Section IV.

\section{Simulation Model And Methodology}

The model used for the current research is FHLR2GCB2G single core shielded and multi stranded cable, conductor area of $35 \mathrm{~mm}^{2}$ and overall diameter of $14.4 \mathrm{~mm}$, from Coroflex manufacturer, especially designed for automotive electric powertrain up to $600 V_{A C} / 1000 V_{D C}$. In Figure 2 a cross-section of the layers of cable is illustrated. This cable employs double-layer shielding regards to the inner and outer shielding layer using respectively tinned copper wire braiding and aluminum foil.

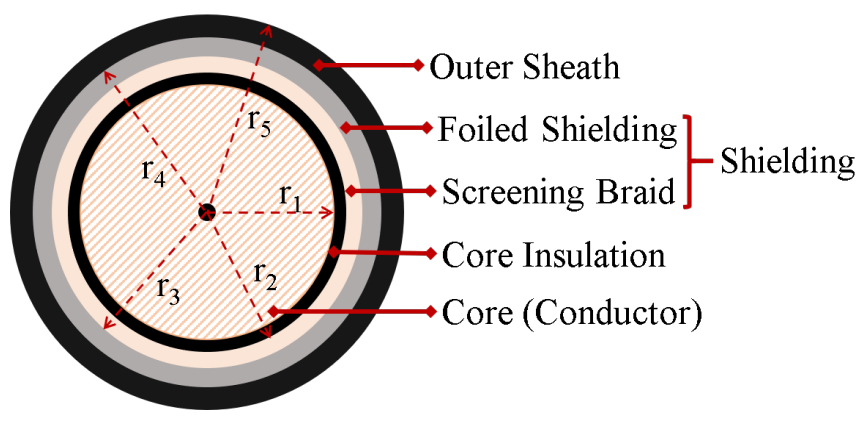

Fig. 2. Cable layers for the model FLHR2GCB2G.

The simulation was performed in a 64-bit PC SO Windows 10 Pro, Intel Core i5-9600 CPU six core, $3.10 \mathrm{GHz}$ clock base frequency, 16GB-RAM. The current method uses the cable geometrical dimensions provided by manufacturer and extracts the transmission line parameters from such model for one cell (1 cm cable length) by using COMSOL version 5.6 (FEA software). Then, they are extended to 100 cells (1-meter long) as an evaluation of frequency response of the cable and it was carried out using LTspice version XVII.

For selection of the segment cell $\Delta l$ of the cable, it was chosen $100 \mathrm{MHz}$ as the operating frequency, once some new in-vehicle networks - communication intended to be used within a vehicle only, not providing communication to others - can reach it e.g. Ultra-Wide Band (UWB) radio technology and Media Oriented Systems Transport (MOST) protocol. Considering (3) and $f_{H O}=100 \mathrm{MHz}$, thus the $\lambda \approx 3 \mathrm{~m}$. However, as the rule of thumb $\Delta l$ must be $1 / 10 \lambda$, i.e. $\Delta l<0.3$ $\mathrm{m}$. The value chosen was $\Delta l=0.01 \mathrm{~m}$. Considering the cable length of $1 \mathrm{~m}$, the entire model will have 100 cells and it will 
be later simulated on SPICE. The values of radii along with cable material specification are shown in Table I, according to [15], [16].

TABLE I

MATERIAL SPECIFICATION OF THE CABLE MODEL.

\begin{tabular}{lccccc}
\hline \multicolumn{1}{c}{ Layer } & Material & $\begin{array}{c}\text { Radius } \\
\mathbf{( m m})\end{array}$ & $\begin{array}{c}\sigma \\
\mathbf{( S / m )}\end{array}$ & $\mu_{r}$ & $\epsilon_{r}$ \\
\hline Core & Copper & $r_{1}=3.338$ & $5.9595 \mathrm{e}+07$ & 1 & 1 \\
Core Insulation & Silicone & $r_{2}=5.250$ & $1.2100 \mathrm{e}-05$ & 1 & 2.7 \\
Screening Braid & Copper & $r_{3}=5.460$ & $5.9595 \mathrm{e}+07$ & 1 & 1 \\
Foiled Shielding & Aluminum & $r_{4}=5.560$ & $3.7736 \mathrm{e}+07$ & 1 & 1 \\
Outer Sheath & Silicone & $r_{5}=7.200$ & $1.2100 \mathrm{e}-05$ & 1 & 2.7 \\
\hline
\end{tabular}

Given the length dimension, however, the conductor used was designed solid rather multi stranded. After designing the model (3D drawing, applying materials to domains and specify the conductivity, permeability and permittivity of the materials), the model was meshed (division of model into several finite elements). In Figure 3 the cable designed in COMSOL is shown after meshing process. It was used 1 $\mathrm{mm}$ and $0.01 \mathrm{~mm}$ for maximum and minimum element sizes, respectively.

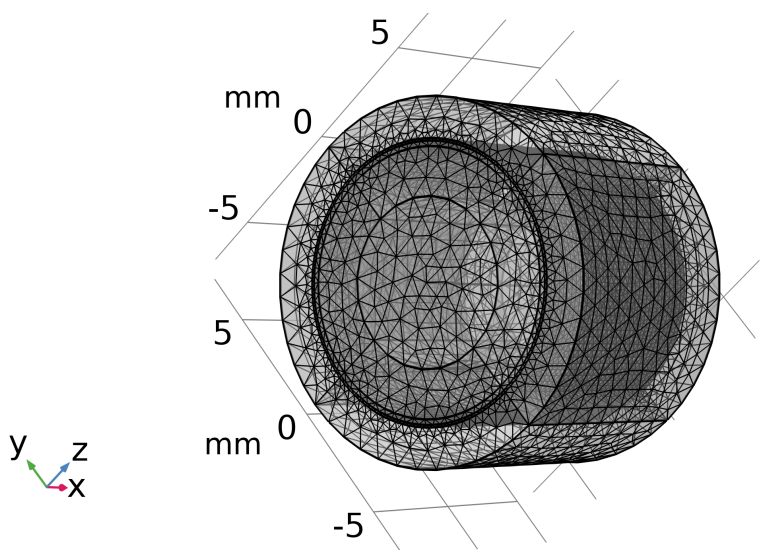

Fig. 3. Model meshing in COMSOL.

Thus, after meshing, the model was simulated with 1A DC or $1 \mathrm{~V} \mathrm{DC}$ as input current and voltage, respectively. The results were obtained through data post-processing.

\section{RESUlts AND Discussion}

The whole time to process to obtain results using 1 cell was $29 \mathrm{~s}$. For the matter of comparison, using the same input level details for $1 \mathrm{~m}$ length, it was found "out of memory" issue, which denotes the computer system has no sufficient RAM memory to process the calculations. In order to overcome this and loosen up the computational burden, the simulation was carried out for $1 \mathrm{~cm}$ cable length and extended to $1 \mathrm{~m}$ using transmission lines theory. The values obtained and physical interface through simulation are described in Table II. The conductance was disregarded due to the overall length of the model.

The propagation delay was found to be $\gamma=5.42 \eta \mathrm{s}$ while characteristic impedance obtained is $Z_{0}=13.89 \Omega$.
TABLE II

VALUES OBTAINED IN SIMULATION FOR $\Delta l=1 \mathrm{CM}$.

\begin{tabular}{lcl}
\hline Parameter & Value & Physical Interface \\
\hline$R$ & $4.7651 \mathrm{E}-06 / \mathrm{cm}$ & Electric Circuits \\
$L$ & $7.5379 \mathrm{E}-10 / \mathrm{cm}$ & Magnetic Fields \\
$C$ & $3.9049 \mathrm{E}-12 / \mathrm{cm}$ & Electrostatics \\
$G$ & - & - \\
\hline
\end{tabular}

The magnetic flux density normal field of the model crosssection Y-Z plane is shown in the Fig. 4. It is possible to see that the flux density reaches its maximum at $49 \mu \mathrm{T}$ in the interface between the conductor and the core insulation. At the boundary of shield it is around $20 \mu \mathrm{T}$ and at the outer sheath it reaches $7 \mu \mathrm{T}$. It means the shielding is acting properly blocking the flux density.

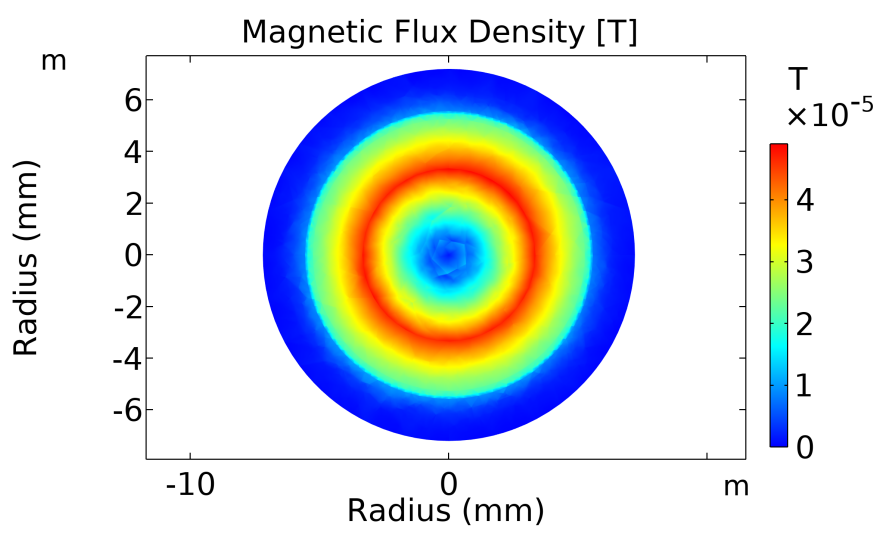

Fig. 4. Magnetic flux density over the cable cross section.

A better view of the flux density is observed in the chart of Fig. 5. This chart shown the flux density over a central measurement line in the cross section of the cable and it is seen the intensity is axial symmetric once it describes the normal vector of flux density. As shown, the flux density reaches its peaks at the conductor-dielectric interface and it is null in conductor center and at the external boundaries between air and the outer sheath, once there is no variation of magnetic field.

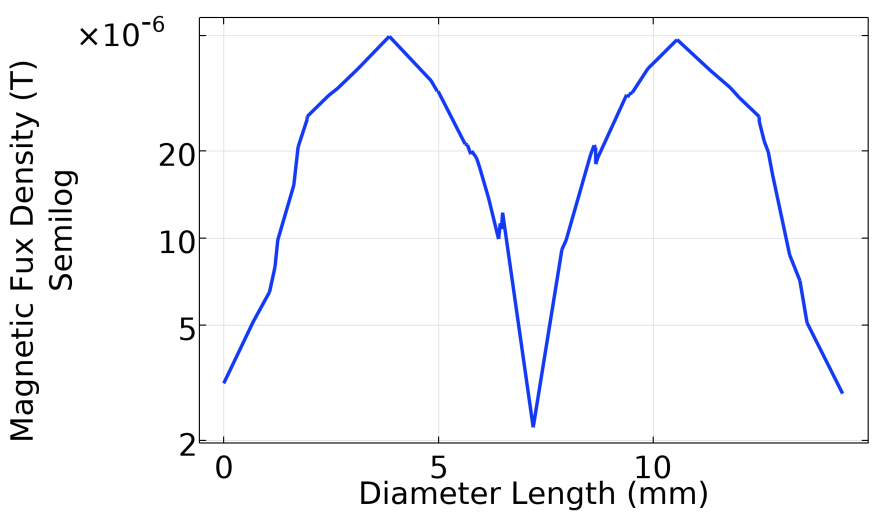

Fig. 5. Flux density intensity line along the cable diameter. 
Once gathered the transmission line parameters, the circuit in LTspice was mounted and simulated, as shown in Fig. 6. It was applied $1 \mathrm{~V}$ AC from $0.1 \mathrm{~Hz}$ to $1 \mathrm{GHz}$ to the circuit as the input voltage. In order to get $\Gamma=0$, the $Z_{0}$ has the same magnitude than $Z_{0}$. In addition, considering (4) and the obtained parameters, the resonance frequency was analytically calculated and reaching the value of $29 \mathrm{MHz}$.

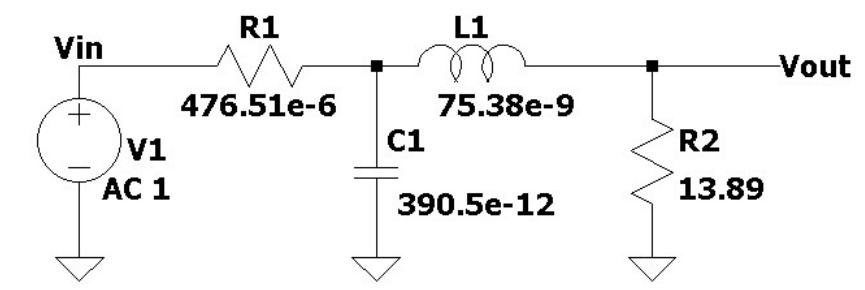

; tran 10m

.ac lin $10000000.1100 e 9$

Fig. 6. LTspice simulation for the circuit parameters.

Thus, firstly the frequency response of the circuit was obtained and the resonance was found to be $21 \mathrm{MHz}$ and, as expected, the frequency response shows an attenuation above it, behaving likewise a low-pass filter. Over the cut-off frequency, the inductive and capacitive reactance will affect the output voltage response.

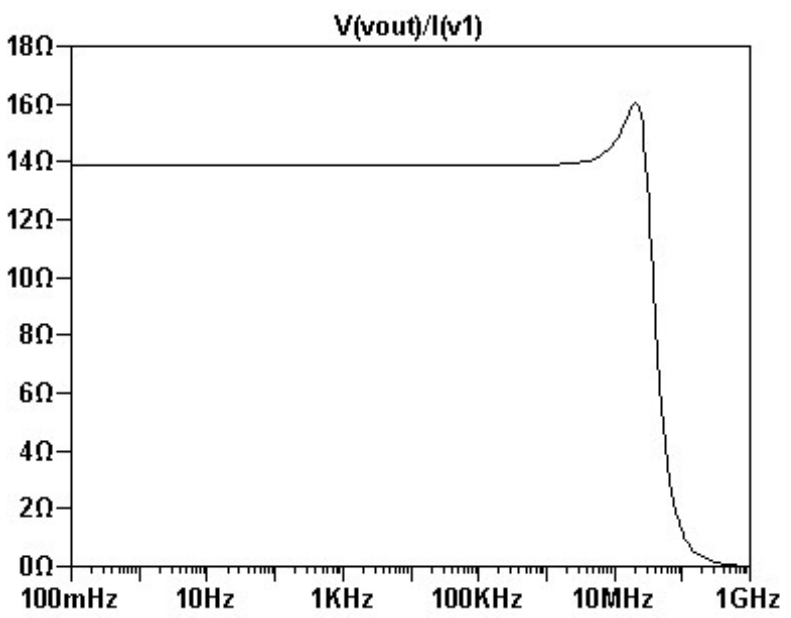

Fig. 7. Transfer impedance for the entire cable (100 cells) in LTspice.

In other words, it can represent an EMC issue if an invehicle communication or a signal cable is located near such power cable and operates around 20-30 MHz.

\section{CONCLUSION}

This paper presented a method used for simulate long cables with detailed specification using FEA and transmission line theory. Through this, the transmission line parameters for a single segment cell $(1 \mathrm{~cm})$ was gathered and the cable extension up to 100 cells ( $1 \mathrm{~m}$ long cable) was done. Then the validation of such method was carried out using SPICE.
This analysis can be especially extended to signal cables and double power vehicular cable to assess in a very detailed way the electromagnetic behavior, shield efficiency and the signal attenuation or interference. It will be the next steps of the current research in a further comparison with experimental evaluation.

\section{ACKNOWLEDGMENT}

The authors would like to thanks the Institute of Automatic Control, Electronics, and Electrical Engineering at the University of Zielona Góra for the facilities and equipment for carrying out the simulations.

\section{REFERENCES}

[1] J. Hu, X. Xu, D. Cao, and G. Liu, "Analysis and optimization of electromagnetic compatibility for electric vehicles," IEEE Electromagnetic Compatibility Magazine, vol. 8, no. 4, pp. 50-55, 2019.

[2] C. Wu, F. Gao, H. Dai, and Z. Wang, "A topology-based approach to improve vehicle-level electromagnetic radiation," Electronics (Switzerland), vol. 8, no. 3, 2019.

[3] Q. Duan, C. Feng, and L. Xia, "Discussion on the technology of high voltage cable for hybrid electric vehicle," Journal of Physics: Conference Series, vol. 1846, no. 1, 2021.

[4] L. Yang, S. Wang, and Y. Zhi, "Near Field Coupling Measurement and Modeling between High Voltage and Low Voltage Cables in Electric Vehicles," 2020 IEEE International Symposium on Electromagnetic Compatibility and Signal/Power Integrity, EMCSI 2020, pp. 404-406, 2020.

[5] H. Loschi, R. Smolenski, P. Lezynski, D. Nascimento, and G. Demidova, "Aggregated conducted electromagnetic interference generated by DC/DC converters with deterministic and random modulation," Energies, vol. 13, no. 14, 2020.

[6] R. Collin, Y. Miao, A. Yokochi, P. Enjeti, and A. Von Jouanne, "Advanced electric vehicle fast-charging technologies," Energies, vol. 12, no. $10,2019$.

[7] Y. Mizutani and O. Weiss, "Pipe shield high-voltage wiring harness," World Electric Vehicle Journal, vol. 5, no. 2, pp. 581-587, 2012.

[8] X. Wu, X. Shi, J. Jia, H. Zhao, and X. Li, "Shield reliability analysisbased transfer impedance optimization model for double shielded cable of electric vehicle," Mathematical Problems in Engineering, vol. 2021, 2021.

[9] J. C. Del-Pino-López, M. Hatlo, and P. Cruz-Romero, "On simplified 3D finite element simulations of three-core armored power cables," Energies, vol. 11, no. 11, 2018.

[10] K. Feldhues, M. Diebig, and S. Frei, "Analysis of the low frequency shielding behavior of high voltage cables in electric vehicles," IEEE International Symposium on Electromagnetic Compatibility, no. 3, pp. 408-413, 2014.

[11] Y. Weens, N. Idir, R. Bausière, and J. J. Franchaud, "Modeling and simulation of unshielded and shielded energy cables in frequency and time domains," IEEE Transactions on Magnetics, vol. 42, no. 7, pp. 1876-1882, 2006.

[12] S. Celozzi, R. Araneo, and G. Lovat, Electromagnetic Shielding. New Jersey: John Wiley \& Sons, 2008, vol. 1.

[13] A. L. Shenkman and M. Zarudi, "Transmission lines," in Circuit Analysis for Power Engineering Handbook. Kluwer Academic Publishers, 1998.

[14] A. Tsaliovich, Cable Shielding for Electromagnetic Compatibility. New York: Chapman \& Hall, 1995.

[15] F. Madidi, G. Momen, and M. Farzaneh, "Dielectric Properties of TiO2/Silicone Rubber Micro- and Nanocomposites," Advances in Materials Science and Engineering, vol. 2018, 2018.

[16] Terry C. Edwards and M. B. Steer, "Appendix B - Material Properties," in Foundations for Microstrip Circuit Design, 2016. 